\title{
EVALUACIÓN DE LA SEGURIDAD ALIMENTARIA Y NUTRICIONAL EN FAMILIAS DEL DISTRITO DE LOS MOROCHUCOS EN AYACUCHO, PERÚ
}

\author{
Susan Pillaca ${ }^{1 a}$, María Villanueva ${ }^{1, b}$
}

RESUMEN

Objetivos. Evaluar la seguridad alimentaria y nutricional en familias del distrito Los Morochucos en Ayacucho, Perú. Materiales y métodos. Estudio basado en interpretación de la información disponible de fuentes oficiales y de encuestas realizadas para levantar la información no registrada en ellas. El muestreo fue estratificado y bietapico considerando 128 familias con al menos un niño menor de 12 años. Las variables fueron el nivel de seguridad alimentaria, percepción familiar sobre su situación de disponibilidad y acceso de sus alimentos, lactancia materna exclusiva, alimentación durante episodios de diarrea, frecuencia de consumo de alimentos, consumo de agua segura, lavado de manos, servicios higiénicos adecuados, estabilidad en el suministro de alimentos, estado nutricional y de salud, desnutrición crónica, anemia y parasitosis en niños de 6 meses a tres años. Resultados. El 39,1\% de familias tiene seguridad alimentaria, $37,5 \%$ inseguridad alimentaria sin hambre, $18,8 \%$ inseguridad alimentaria con hambre, y $4,7 \%$ inseguridad alimentaria con hambre severa. Tienen aceptable consumo de calorías, pero no tienen una dieta balanceada. No cuentan con agua clorada. Durante los episodios de diarrea, $72 \%$ de madres alimenta al niño con mates o caldos. Se perdieron gran cantidad de sus cosechas el año 2011. Los niños de 6 meses a tres años presentan $34,5 \%$ de desnutrición crónica, 42,4\% de anemia y $61,6 \%$ parasitosis. Conclusiones. La inseguridad alimentaria y nutricional en este distrito de Ayacucho es considerable debiéndose priorizar la ejecución de proyectos de inversión con el fin de contribuir el desarrollo de la comunidad.

Palabras clave: Seguridad alimentaria; Desnutrición; Lavado de manos; Lactancia materna; Anemia (fuente: DeCS BIREME).

\section{EVALUATION OF FOOD AND NUTRITION SECURITY IN FAMILIES IN THE DISTRICT OF LOS MOROCHUCOS IN AYACUCHO, PERU}

\begin{abstract}
Objectives. To evaluate food and nutrition security in families of Los Morochucos district in Ayacucho, Peru. Materials and methods. A study based on interpretation of available information from official sources and surveys carried out to gather information not recorded in these sources. Sampling was stratified and two-staged comprising 128 families with at least one child under 12 years. The variables were the level of food security, family perception of their situation of availability of and access to food, exclusive breastfeeding, feeding during episodes of diarrhea, frequency of food consumption, consumption of safe water, hand washing, proper toilets, stable food supply, nutrition and health status, chronic malnutrition, anemia and parasitic infections in children aged 6 months to three years. Results. $39.1 \%$ of families had food security, $37.5 \%$ were food insecure without hunger, $18.8 \%$ had food insecurity with hunger, and $4.7 \%$ had food insecurity with severe hunger. They had acceptable calorie intake, but did not have a balanced diet. There was no chlorinated water. During the episodes of diarrhea, $72 \%$ of mothers fed the child with mate (tea) or broth. A great quantity of their crops were lost in 2011 . Of children 6 months to three years, $34.5 \%$ presented chronic malnutrition, $42.4 \%$ had anemia and $61.6 \%$ had parasites. Conclusions. Food and nutrition insecurity in this district of Ayacucho is considerable and there should be prioritization of the implementation of investment projects with the aim of contributing to the development of the community.
\end{abstract}

Key words: Food security; Malnutrition; Hand disinfection; Breast feeding, Anemia (source: MeSH NLM).

\section{INTRODUCCIÓN}

La seguridad alimentaria es el derecho que las personas tienen a disponer en todo momento de una suficiente cantidad y calidad de alimentos que le permitan una vida saludable y activa ${ }^{(1)}$. En el Perú, el año 2004 se aprobó la Estrategia Nacional de Seguridad Alimentaria 20042015, cuya misión es brindar mejores condiciones para la disponibilidad, acceso, uso y estabilidad de alimentos, para garantizar la seguridad alimentaria y nutricional de la población peruana, priorizando los grupos vulnerables y en pobreza extrema ${ }^{(2)}$. 
Estudios de evaluación del impacto de intervenciones en seguridad alimentaria como el desarrollado por la ONG CARE en algunos distritos de Perú, muestra un importante impacto en la reducción de la desnutrición crónica y en el incremento del gasto destinado a los alimentos, manifestando la efectividad de este tipo de programas ${ }^{(3)}$, ya que se trabaja de una manera social y productiva teniendo una mejor sostenibilidad en el tiempo a diferencia de los programas asistencialistas que no muestran mejoras en los indicadores nutricionales ${ }^{(4)}$.

Estudiar las consecuencias nutricionales de inseguridad alimentaria es un problema sumamente complejo cuya solución involucra diferentes sectores. Se ha considerado evaluar en este estudio, el estado de los diferentes aspectos de las dimensiones de la seguridad alimentaria y nutricional en un distrito de Ayacucho, de esta manera se podrá plantear mejores proyectos de inversión económica, social y de salud priorizados de acuerdo a sus propias particularidades, con el fin de contribuir al desarrollo de la familia y de la comunidad.

Las dimensiones estudiadas son: seguridad alimentaria, donde se ve la disponibilidad de alimentos, la que debe asegurar la existencia de alimentos de forma estable e inocua durante todo el año en el mercado local, como resultado tanto de la producción interna como de productos industrializados y sus reservas, también está asociado al acceso a los alimentos, que depende tanto de la oferta (disponibilidad) en el mercado, del acceso económico en forma permanente y oportuna. La parte nutricional que corresponde al consumo apropiado, cantidad y calidad de alimentos, de acuerdo a los requerimientos y los factores de utilización biológica, condiciones de higiene y saneamiento que aseguren la salud el que repercute en la biodisponibilidad de los nutrientes $y$, por ende, en el estado nutricional. El objetivo de la investigación fue evaluar la seguridad alimentaria y nutricional en familias del distrito de Los Morochucos en Ayacucho, Perú

\section{MATERIALES Y MÉTODOS}

\section{DISEÑO Y POBLACIÓN DEL ESTUDIO}

Estudio de tipo transversal y descriptivo basada en información de fuentes oficiales como el Instituto Nacional de Estadística e Informática (INEI), el Centro Nacional de Alimentación y Nutrición (CENAN), el Fondo de Las Naciones Unidas Para la Infancia (UNICEF); la Municipalidad de Los Morochucos (MDL) y encuestas para recoger información no consignada en las fuentes oficiales. La aplicación de las encuestas, se realizó entre noviembre y diciembre de 2011.
Se seleccionaron familias con al menos un niño menor de 12 años residentes en alguna de las doce comunidades del distrito de Los Morochucos que son: Munaypata, Satica, Cusibamba, Chanquil, Calvario, Juscaymarca, Cuchucancha, Buenavista, Pampa Cangallo, Jatumpampa, Pilpicancha y Hualchancca. Se escogieron familias que tuviesen hijos menores de doce años porque actualmente el estado viene atendiendo a este grupo de niños con diferentes proyectos.

\section{SELECCIÓN DE LA MUESTRA}

El muestreo utilizado fue probabilístico, estratificado, en una sola etapa para el caso de centros poblados, y bietapico para el caso de la población dispersa. La estratificación fue por centros poblados que en total son ocho; considerando las comunidades dispersas como otro estrato, haciendo un total de nueve estratos. El tamaño de la muestra fue calculado a través de la fórmula para estimación de proporciones, con un error previsto de $9 \%$ y una tasa de no respuesta del $20 \%$. En total se encuestaron 128 madres de familia, de las cuales para cada estrato se encuestó una cantidad de viviendas proporcional al tamaño del estrato.

Para los centros poblados se escogió una vivienda de inicio de manera aleatoria, las demás viviendas se seleccionaron de manera sistemática. Para la población dispersa en la primera etapa se seleccionó aleatoriamente cuatro comunidades (conglomerados) y el tamaño de muestra para cada conglomerado fue proporcional al tamaño del conglomerado. En la segunda etapa se seleccionó las viviendas dentro de cada conglomerado, para ello, primero se determinó una vivienda de inicio, seleccionada de manera aleatoria simple, luego se procedió a seleccionar las siguientes de manera sistemática a partir de la vivienda de inicio.

\section{INDICADORES PARA CADA COMPONENTE Y FUENTES DE VERIFICACIÓN}

Se aplicaron dos formatos de encuesta una, del Departamento de Agricultura de Estados Unidos que fue adaptada para el Perú (5), la que asigna un valor de seguridad alimentaria a la población, y para analizar los factores involucrados en el componente de consumo apropiado y utilización biológica se realizó una encuesta estructurada. Se obtuvo también información sobre la estabilidad en el suministro de alimentos, debido a que en esta zona la mayor parte de sus ingresos son obtenidos de la actividad agrícola y ganadera. 


\section{NIVEL DE SEGURIDAD ALIMENTARIA}

Se aplicó una encuesta en el hogar para evaluar la percepción de las familias sobre la disponibilidad y acceso a sus alimentos durante un año, la cual permite determinar el porcentaje de familias que cuenta con seguridad alimentaria, o inseguridad alimentaria con hambre moderado o hambre severo ${ }^{(5)}$.

\section{CONSUMO APROPIADO}

De la información previamente recabada por la Municipalidad Distrital de Los Morochucos se tomaron datos de hábitos de lactancia materna exclusiva y alimentación durante y después de las enfermedades diarreicas (2010). Se tomó en cuenta estos factores debido a que están relacionados en mayor parte al consumo apropiado de alimentos.

\section{UTILIZACIÓN BIOLÓGICA}

Se consideran a los principales factores que afectan la salud y, por ende, la buena utilización biológica de los alimentos como son: servicios de agua segura en el hogar y disponibilidad de servicios higiénicos adecuados según INEI; la información sobre el lavado de manos y las formas de eliminar la basura se obtuvo de la encuesta estructurada desarrollada en los hogares.

\section{ESTABILIDAD EN EL SUMINISTRO}

Pérdidas en producción debido a heladas, sequias, inundaciones y otros factores obtenidos mediante la encuesta en los hogares.

\section{SITUACIÓN NUTRICIONAL Y DE SALUD}

Como los principales factores considerados problemas de salud pública según ENDES 2011-2012, se tomaron en cuenta: desnutrición crónica en niños menores de 3 años (MDL 2010), anemia en niños de 6 meses a tres años (MDL, 2010) y parasitosis en niños de 6 meses a tres años (MDL, 2010).

\section{ANÁLISIS ESTADÍSTICO}

Las encuestas realizadas se digitaron en una base de datos del software estadístico SPSS v. 18, para obtener los resultados en porcentajes para cada indicador e intervalos de confianza al $95 \%$, con las frecuencias respectivas; el análisis final se hizo de una manera reflexiva y descriptiva.
Tabla 1. Nivel de seguridad alimentaria en familias del distrito de Morochuco, Ayacucho

\begin{tabular}{lccc}
\hline \multicolumn{1}{c}{ Nivel } & N & \% & (IC 95\%) \\
\hline Seguridad alimentaria & 50 & 39,1 & $(30,8-47,3)$ \\
$\begin{array}{l}\text { Inseguridad alimentaria sin } \\
\text { hambre }\end{array}$ & 48 & 37,5 & $(29,3-45,7)$ \\
$\begin{array}{l}\text { Inseguridad alimentaria con } \\
\text { hambre moderada }\end{array}$ & 24 & 18,8 & $(12,2-25,3)$ \\
$\begin{array}{l}\text { Inseguridad alimentaria con } \\
\text { hambre severa }\end{array}$ & 6 & 4,7 & $(1,1-8,3)$ \\
\hline
\end{tabular}

\section{CONSIDERACIONES ÉTICAS}

El estudio contó con la aprobación del Comité de Ética del Instituto de Investigación Nutricional. Al momento de llevarse a cabo las entrevistas a las madres de familia se les explicó sobre el trabajo y se hizo firmar la hoja de consentimiento informado; en el caso de personas analfabetas se tomó su huella digital del dedo índice derecho. La participación fue voluntaria y la información se trató con confidencialidad.

\section{RESULTADOS}

\section{EVALUACIÓN DE LA SEGURIDAD ALIMENTARIA}

En el distrito de Los Morochucos existe $39,1 \%$ de familias con seguridad alimentaria, $37,5 \%$ con inseguridad alimentaria sin hambre, $18,8 \%$ con inseguridad alimentaria con hambre moderada y $4,7 \%$ con inseguridad alimentaria con hambre severa (Tabla 1). Las comunidades en las que hay familias con inseguridad alimentaria con hambre severa son Pilpicancha $(16,2 \%)$, Cusibamba $(14,3 \%)$, población dispersa $(5,9 \%)$ y Pampa $(2,8 \%)$.

\section{CONDICIONES DE CONSUMO APROPIADO}

Según los resultados de la $\mathrm{MDL}^{(6)}$, a nivel distrital $96,4 \%$ de madres practica lactancia materna exclusiva. Durante episodios de diarrea el $55,9 \%$ de madres alimenta a su niño con agüitas y mates, y $16,9 \%$ con sopas y caldos y $8,1 \%$ con papillas o segundos. El $98,4 \%$ de familias tiene aceptable consumo de calorías en alimentos ${ }^{(7)}$. El 3,9\% de las familias consume frutas diariamente, la mayoría la consume una vez a la semana, en general, las verduras son consumidas en sopas o segundos $y$ poco como ensalada de verduras (Tabla 2).

\section{CONDICIONES DE UTILIZACIÓN BIOLÓGICA}

El $8,6 \%$ toma agua hervida y a veces cruda. El $74,2 \%$ de madres se lava las manos antes de preparar los 
Tabla 2. Condiciones de consumo apropiado

\begin{tabular}{|c|c|c|c|}
\hline Nivel & $\mathbf{N}$ & $\%$ & (IC 95\%) \\
\hline \multicolumn{4}{|l|}{$\begin{array}{l}\text { Lactancia materna exclusiva } \\
(\mathrm{N}=251)\end{array}$} \\
\hline $\begin{array}{l}\text { Lactancia maternas exclusiva hasta } \\
\text { los } 6 \text { meses }\end{array}$ & 242 & 96,4 & $(94,2-98,6)$ \\
\hline $\begin{array}{l}\text { Lactancia materna menor a los } 6 \\
\text { meses }\end{array}$ & 9 & 3,6 & $(1,4-5,8)$ \\
\hline \multicolumn{4}{|c|}{ Practicas adecuadas de alimentación durante EDA ( $N=136)$} \\
\hline Alimentación con agüitas y mates & 76 & 55,9 & $(47,8-64,0)$ \\
\hline Alimentación con sopas y caldos & 23 & 16,9 & $(10,8-23,0)$ \\
\hline Alimentación con segundo o papilla & 11 & 8,1 & $(3,6-12,5)$ \\
\hline Alimentación con lactancia materna & 27 & 19,9 & $(13,3-26,4)$ \\
\hline \multicolumn{4}{|c|}{ Frecuencia de consumo de alimentos $(\mathrm{N}=128)$} \\
\hline $\begin{array}{l}\text { Consumo aceptable en calorías de } \\
\text { alimentos }\end{array}$ & 126 & 98,4 & $(96,3-100)$ \\
\hline $\begin{array}{l}\text { Consumo limite en calorías de } \\
\text { alimentos }\end{array}$ & 2 & 1,6 & $(0,0-3,7)$ \\
\hline Consumo diario de fruta $(\mathrm{N}=128)$ & 5 & 3,9 & $(0,6-7,2)$ \\
\hline
\end{tabular}

alimentos, 48,4\% antes de comer, $41,4 \%$ antes de darle de comer a su hijo, y $37,5 \%$ después de ir al baño. El porcentaje de familias que se lava las manos siempre con agua y jabón fue $7,8 \%$. Solo $8,0 \%$ de los hogares cuenta con red pública de desagüe, y $24,1 \%$ no tiene ningún tipo de servicio higiénico, según el INEI (2007) ${ }^{(8)}$. $40,6 \%$ de familias elimina la basura quemándola, 37,5\% la entierra y solo el 16,4\% tiene servicio de camión recolector (Tabla 3 ).

Tabla 3. Factores que influyen en la utilización biológica de los alimentos

\begin{tabular}{lccc}
\hline \multicolumn{1}{c}{ Factores } & N & \% & (IC 95\%) \\
\hline Consumo de agua segura (N=128) & & & \\
Tomo agua hervida siempre & 117 & 91,4 & $(86,7-96,1)$ \\
\hline Tomo agua hervida y a veces cruda & 11 & 8,6 & $(3,9-13,3)$ \\
Lavado de manos (N=128) & & & \\
Antes de preparar los alimentos & 95 & 74,2 & $(66,8-81,6)$ \\
Antes de comer & 62 & 48,4 & $(40-56,9)$ \\
Antes de darle de comer a mi hijo & 53 & 41,4 & $(33,1-48,7)$ \\
Después de ir al baño & 48 & 37,5 & $(29,3-45,7)$ \\
Como se lava las manos (N=128) & & & \\
Con agua y jabón siempre & 10 & 7,8 & $(3,3-12,3)$ \\
Con agua y jabón; y a veces solo con & 118 & 92,2 & $(87,7-96,7)$ \\
agua & & & \\
Servicio higiénico (N=2253)* & 180 & 8,0 & $(8,0-8,0)$ \\
Red pública de desagüe & 50 & 2,2 & $(2,2-2,2)$ \\
Pozo séptico & 1480 & 65,7 & $(65,7-65,7)$ \\
Pozo ciego / letrina & 543 & 24,1 & $(24,1-24,1)$ \\
No tiene & & & \\
Manera de eliminar la basura & & & \\
(N=128) & 21 & 16,4 & $(10,2-22,7)$ \\
Camión recolector & 7 & 5,5 & $(1,6-9,3)$ \\
En la calle o campo & 52 & 40,6 & $(32,3-48,9)$ \\
Quemándola & 48 & 37,5 & $(29,3-45,7)$ \\
\hline Enterrándola & & &
\end{tabular}

*Según INEI 2007
Tabla 4. Porcentaje de familias que declaran haber perdido sus cultivos

\begin{tabular}{lccc}
\hline \multicolumn{1}{c}{ Cultivo } & N & $\%$ & (IC 95\%) \\
\hline Papa $(\mathrm{N}=106)$ & 96 & 90,6 & $(85,1-96,0)$ \\
Maíz $(\mathrm{N}=64)$ & 62 & 96,9 & $(92,6-100)$ \\
Arveja $(\mathrm{N}=58)$ & 49 & 84,5 & $(75,2-93,8)$ \\
Haba $(\mathrm{N}=59)$ & 48 & 81,4 & $(71,5-91,2)$ \\
Quinua $(\mathrm{N}=52)$ & 37 & 71,2 & $(58,9-83,4)$ \\
Trigo (N=36) & 20 & 55,6 & $(39,2-71,9)$ \\
\hline
\end{tabular}

\section{ESTABILIDAD EN EL SUMINISTRO DE ALIMENTOS}

Las condiciones de estabilidad en el suministro de alimentos para el año 2011 fueron deficientes ya que se perdieron gran parte de las cosechas debido a las alteraciones climáticas como las heladas y granizo que acompañaron a las lluvias (Tabla 4).

\section{DESNUTRICIÓN CRÓNICA}

Según los resultados de la MDL (10), la desnutrición crónica en niños menores de tres años fue de $34,5 \%$ y para desnutrición global $6,1 \%$. Las comunidades de Munaypata $(50 \%)$, Cuchucancha $(50 \%)$, Cusibamba $(47,4 \%)$ y Pilpicancha $(47,1 \%)$ son las que presentan el mayor porcentaje de desnutrición crónica. Las comunidades de Buenavista (25\%), Pampa (25\%), Satica $(26,9 \%)$ y Llumchicancha $(27,3 \%)$ presentan el menor porcentaje de desnutrición crónica.

\section{ANEMIA EN NIÑOS DE 6 MESES A TRES AÑOS}

Según los resultados de la MDL (11) El porcentaje de anemia en niños de 6 meses a tres años fue 12,1\% para anemia moderada (7-9,9 mg/dL) y $30,3 \%$ de anemia leve (10-10,9 mg/dL). La comunidad de Satica $(61,5 \%)$ es la que presenta el mayor número de casos de anemia.

\section{PARASITOSIS INTESTINAL}

Según los resultados de la MDL, $62 \%$ de niños de 6 meses a 3 años tiene algún tipo de parasitosis intestinal (11). Las comunidades de Pampa (87,9\%), Hualchancca $(63,2 \%)$ y Llumchicancha $(53,6 \%)$ son las que presentan el mayor porcentaje.

\section{DISCUSIÓN}

El porcentaje considerable de inseguridad alimentaria con hambre moderada y con hambre severa durante el 2011 puede deberse a que esta zona fue afectada por el clima, como está ocurriendo en muchos lugares 
debido al cambio climático ${ }^{(12)}$. Según la evaluación de estabilidad en el suministro se perdieron la mayor parte de los cultivos como maíz, arveja, haba y papa, la mayor parte de los cultivos son en secano y, como las lluvias llegaron muy tarde y fueron muy intensas, acompañadas además de heladas y granizos, la cosecha para el año 2011 no fue buena, esta situación repercute en la seguridad alimentaria ${ }^{(13)}$. Además, la pobreza total es del $59 \%$ y la pobreza extrema es de $26 \%{ }^{(14)}$, lo cual disminuye el acceso de los alimentos y afecta el estado nutricional ${ }^{(15)}$

Se encontró como positivo el alto el porcentaje de madres que practica lactancia materna exclusiva, lo que protege de la desnutrición a los menores de 6 meses, el MINSA, incorporó la estrategia de seguridad alimentaria a través de los lineamientos nutricionales de continuar promoviendo la lactancia materna exclusiva como práctica saludable y como único alimento para el niño durante los primeros 6 meses de vida, desalentando el uso de agüitas, infusiones u otros alimentos, para así contribuir con el desarrollo integral de los niños ${ }^{(16)}$. La lactancia materna exclusiva y la alimentación complementaria apropiada son dos prácticas importantes para evitar la desnutrición en los niños ${ }^{(17) .}$

Hay una situación muy crítica en la alimentación durante episodios de diarrea ya que mayor parte de las madres alimenta a sus niños con sopas y caldos, el niño en esta situación requiere más nutrientes, especialmente de proteínas ${ }^{(18)}$ y este tipo de alimentación no es nada favorable para la recuperación del niño. En el distrito, las madres siguen pensando aún que las sopas o caldos son más nutritivos que el plato de segundo, por lo que la desnutrición es más frecuente en niños entre 8 y 24 meses de edad, y es consecuencia de problemas de prácticas inapropiadas de alimentación complementaria ${ }^{(17)}$.

Si bien es cierto se ha incrementado considerablemente la tenencia de biohuertos, las verduras son básicamente utilizadas para aderezos y guisos mas no así para la ensalada, es aun más crítico el consumo de frutas ya que solo $3,9 \%$ las consume diariamente, la mayor parte de la población las consume una vez a la semana por lo que el aporte de micronutrientes es deficiente, esta situación es uno de los factores que influye en la desnutrición (19). Las frutas y verduras ejercen un efecto protector para la salud ${ }^{(20)}$, y en poblaciones pobres puede lograrse un incremento en el consumo de frutas y verduras a través de estrategias educativas, sin embargo, el acceso limitado a equipos de transformación y conservación de alimentos es determinante del resultado final (21). La OMS ha promocionado el programa cinco al día en todo el mundo para incrementar el consumo de frutas y verduras, es preocupante la poca frecuencia de consumo por lo que se tiene que intervenir con diferentes programas.

Para la buena utilización biológica de los alimentos uno de los componentes importantes es la calidad del agua (22) y en el distrito no se cuenta con agua clorada, a pesar que en la comunidad hay una organización responsable para la cloración y mantenimiento de los canales de agua, esta tiene débil participación. Un porcentaje significativo de personas toma agua cruda, este porcentaje de familias es más vulnerable a tener niños con diarreas y desnutrición (23).

El lavado de manos debe ser siempre con agua y jabón después de los momentos críticos ${ }^{(24)}$, sin embargo, las prácticas de lavado de manos son deficientes ya que aunque el $74,2 \%$ de madres se lava las manos antes de preparar los alimentos, solo $37,5 \%$ lo hace después de ir al baño. El lavado de manos es una medida sencilla y barata que reduce las enfermedades diarreicas ${ }^{(25)}$. Por otro lado, al igual que otras áreas con pobreza extrema del Perú los servicios higiénicos son deficientes ${ }^{(9)}$, lo que afecta la buena utilización biológica de los alimentos ${ }^{(22)}$ ya que es una de las condiciones que favorece al desarrollo de los parásitos intestinales, que en este distrito es alta; asunto preocupante puesto que es uno de los factores que puede generar desnutrición ${ }^{(26,31)}$.

Un alto porcentaje de familias quema la basura, contribuyendo a la contaminación ambiental, y los efectos negativos que puede tener hacia la salud ${ }^{(27)}$. Sin embargo, en algunas comunidades rurales del distrito Los Morochucos algunas ONG han venido trabajando con el sistema de pozo de basura que permite un mejor manejo de los desechos.

La seguridad alimentaria y nutricional puede influir en el estado nutricional, a nivel distrital es considerable la desnutrición crónica en menores de tres años, que presentan valores, superiores al promedio nacional que es de $19,5 \%$ en niños menores de cinco años, la desnutrición crónica tiene efectos negativos inmediatos así como mayor probabilidad de ocurrencia de enfermedades $y$, a largo plazo, podría afectar el desempeño escolar, la capacidad de trabajo, y el aumento de costos y perdidas económicas a la familia y la sociedad (28,29). La suplementación con multimicronutrientes es una alternativa para disminuirla ${ }^{(30)}$.

En conclusión la inseguridad alimentaria y nutricional en el distrito Los Morochucos es considerable y se puede apreciar entre los principales factores que la condicionan a las pérdidas de cosechas por motivos 
climáticos, siendo necesario revisar lo referente a gestión de riesgos y defensa civil, e intervenciones para proteger los cultivos de las inclemencias del clima. Con respecto al consumo de alimentos y a su utilización biológica, se aprecian graves deficiencias, debidas no solo a la falta de acceso económico, sino de educación alimentaria. Las condiciones sanitarias son muy deficientes, tanto en la disponibilidad de agua segura como de las condiciones de eliminación de desechos, lo que interfiere con las condiciones generales de salud de la población y, por ende, de la utilización biológica de los alimentos. Hay mucho trabajo sanitario pendiente en cuanto a vigilancia y monitoreo de la cloración del agua, sensibilización de la población con campañas de lavado de manos, incrementar la tenencia de letrinas en áreas rurales y capacitar en la forma de desinfectar y realizar la limpieza de las mismas.

La información del estado de cada una de las dimensiones de la seguridad alimentaria estudiados en esta comunidad permitirá priorizar proyectos específicos en cada uno de los sectores involucrados que mejoren su situación, así se podrá garantizar una niñez saludable y continuar con el desarrollo de la comunidad.

Agradecimientos: al comité de ética del Instituto de Investigación Nutricional, por el asesoramiento y aprobación de la hoja de consentimiento informado. A Mg Sc Celso Gonzales Chavesta de la Universidad agraria La Molina, por el asesoramiento estadístico. Asimismo, a las personas entrevistadas del Distrito de Los Morochucos por el tiempo e información compartida.

Contribuciones de autoría: SP y MV ha participado en la concepción, diseño del artículo. Además, SP ha participado en la recolección y obtención de resultados, análisis e interpretación de datos y en la redacción del artículo, y MV participo en la revisión crítica del artículo y aprobación de su versión final.

Fuentes de financiamiento: el estudio fue autofinanciado.

Conflictos de interés: los autores declaran no tener conflictos de interés en la publicación de este artículo.

\section{REFERENCIAS BIBLIOGRÁFICAS}

1. Organización de las Naciones Unidas para la Alimentación y la Agricultura. Indicadores de la seguridad alimentaria [Internet]. Roma: FAO; 2014 [citado el 10 de noviembre de 2014]. Disponible en: http://goo.gl/ysbGa0

2. TCP/RLA/2909. Estrategia nacional de seguridad alimentaria en el Perú 2004- 2015 [Internet]. Lima 1112 de octubre. Gobierno del Perú; 2004 [citado el 18 de mayo de 2011]. Disponible en: http://goo.gl/kk865r

3. Rojas C, Flores R, Céspedes R. Resultados de un programa de seguridad alimentaria en la reducción de la desnutrición crónica y sus factores causales en niños peruanos. Rev Peru Med Exp Salud Publica. 2007;24(2):111-20.

4. Vásquez E, Riesco G. Los programas sociales que 'alimentan ' a medio Perú. En: Portocarrero F, ed. Políticas sociales en el Perú: nuevos aportes. Lima: Red para el Desarrollo de las Ciencias Sociales en el Perú. 2000. p. 89-151.

5. Vargas S, Penny M. Measuring food insecurity and hunger in Peru: a qualitative and quantitative analysis of an adapted version of the USDA's Food Insecurity and Hunger Module. Public Health Nutr. 2010
Oct;13(10):1488-97. doi: 10.1017/ S136898000999214X

6. Municipalidad Distrital de Los Morochucos. Informe final de línea de base situación nutricional y conocimientos en alimentación, nutrición y salud en niños menores de 5 años del distrito de Los Morochucos. Ayacucho: MDLM; 2010.

7. Programa Mundial de Alimentos. Manual para la evaluación de la seguridad alimentaria en emergencias. Servicios de análisis de la seguridad alimentaria [Internet]. 2da. ed. Roma: PMA; 2009 [citado el 22 de junio de 2011]. Disponible en: http:// documents.wfp.org/stellent/groups/ public/documents/manual_guide_ proced/wfp203216.pdf

8. Instituto Nacional de Estadística e Informática. Sistema de consulta de datos de centros poblados y población dispersa [CD-ROM]. Lima: INEI; 2007.

9. Velásquez J, Solís L, Vigo W, Rosas A, Giusti P, Fernández P, et al. Evaluación de las prácticas de cuidado materno infantil en áreas con pobreza extrema del Perú, 2012. Rev Peru Med Exp Salud Publica. 2014;31(2):243-53.
10. Municipalidad Distrital de Los Morochucos. Evaluación nutricional de niños y niñas menores de 3 años para determinar la prevalencia de la desnutrición crónica infantil del distrito de Los Morochucos, provincia de Cangallo- Ayacucho; 2012. Ayacucho: MDLM; 2012.

11. Municipalidad Distrital de Los Morochucos. Línea de base de hemoglobina y parasitosis en niños (as) menores de 3 años del distrito de Los Morochucos; 2011. Ayacucho: MDLM; 2011.

12. Feo O, Solano E, Beingolea L, Aparicio M, Villagria M, Prieto M, et al. Cambio climático y salud en la región andina. Rev Peru Med Exp Salud Publica. 2009;26(1):83-93.

13. Velásquez, A. Efectividad de programas de reducción de la pobreza en la nutrición infantil y los determinantes económicos de la desnutrición en países de escasos recursos. Rev Peru Med Exp Salud Publica. 2009;26(4):478-93.

14. Instituto Nacional de Estadística e Informática. Dirección Técnica de Demografía e Indicadores Sociales. Mapa de pobreza provincial y distrital 2009. Lima: INEI; 2010. 
15. Alcázar L, Ocampo D, Huamán-Espino L, Aparco J. Impacto económico de la desnutrición crónica, aguda y global en el Perú. Rev Peru Med Exp Salud Publica. 2013;30(4):569-74.

16. Instituto Nacional de Salud. Lineamientos de nutrición materna infantil del Perú [Internet]. Lima: INS; 2004 [citado el 4 de julio de 2008]. Disponible en: http://bvsalud.org/ portal/resource/es/rep-177120

17. Mamani R. Efectividad del programa educativo "niño sano y bien nutrido" para mejorar los conocimientos, actitudes y prácticas en seguridad nutricional aplicado a madres con niños menores de 1 año del centro de salud Moyopampa, Chosica-Lima. Revista Científica de EnfermeríaRECIEN. 2008.

18. Checkley W, Epstein LD, Gilman RH, Cabrera L, Black RE. Effects of acute diarrhea on linear growth in Peruvian children. Am J Epidemiol. 2003 Jan 15;157(2):166-75.

19. Hernán C. Malnutrición de micronutrients. Estrategia de prevención y control. Colomb Medica. 2001;32(2)01:95-8.

20. Palomo I, Gutiérrez M, Astudillo L, Rivera C, Torres C, Guzmán L, et al. Efecto antioxidante de frutas y hortalizas de la zona central de Chile. Rev Chil Nutr. 2009 Jun;36(2):152-8.

21. Prada G, Dubeibe-Blanco L, Herrán O, Herrera-Anaya M. Evaluación del impacto de un ensayo comunitario sobre el consumo de frutas y verduras en Colombia. Salud Publica Mexico. 2007;49(1):11-9.

22. Oenema S. La seguridad alimentaria en los hogares [Internet]. Roma: Food and Agriculture Organization of the United Nations; 2001 [citado el 13 de mayo de 2011]. Disponible en: http:// www.bvsde.paho.org/bvsacd/cd65/ SeguridadAlimentaria.pdf

23. Miranda M, Aramburú A, Junco J, Campos M. Situación de la calidad del agua para consumo en hogares de niños menores de cinco años en Perú, 2007-2010. Rev Peru Med Exp Salud Publica. 2010;27(4):506-11.

24. Perú, Ministerio de Salud. Con manos limpias niños sanos [Internet]. Lima: MINSA; 2008 [citado el 8 de enero de 2013]. Disponible en: http://www.minsa.gob.pe/portada/ Especiales/2010/lavadodemanos/pdf/ Gigantrograf\%C3\%ADa-6x9.pdf

25. Ejemot RI, Ehiri JE, Meremikwu MM, Critchley JA. Hand washing for preventing diarrhoea. Cochrane Database Syst Rev. 2008 Jan 23;(1):CD004265. doi: 10.1002/14651858.CD004265.pub2.

26. Devera R, Mago Y, Rumhein F. Parasitosis intestinal y condiciones sociosanitarias en niños de una comunidad rural del Estado Bolivar, Venezuela. Rev Biomed. 2006;17(4):311-3.

27. Atencio R, Reyes-López J, GuevaraGarcía J. Evaluación de riesgo ambiental en un tiradero con quema de basura. Rev Int Contam Ambie. 2013;29 (Sup 3):107-17.
28. Black RE, Allen LH, Bhutta ZA, Caulfield LE, de Onis M, Ezzati M, et al. Maternal and child undernutrition: global and regional exposures and health consequences. Lancet. 2008 Jan 19;371(9608):243-60. doi: 10.1016/ S0140-6736(07)61690-0.

29. De-Regil LM, Suchdev PS, Vist GE, Walleser S, Peña-Rosas JP. Home fortification of foods whit multiple micronutrient powders for health and nutrition in children under two years of age. Evid Based Child Health. 2013 Jan;8(1):112-201. doi: 10.1002/ ebch. 1895 .

30. Munayco C, Ulloa-Rea M, MedinaOsis J, Lozano-Revollar C, Tejada V, Castro-Salazar C. Evaluación del impacto de los multimicronutrientes en polvo sobre la anemia infantil en tres regiones andinas del Perú. Rev Peru Med Exp Salud Publica. 2013;30(2):229-34.

31. Ordoñez L, Angulo E. Desnutrición y su relación con parasitismo intestinal en niños de una población de la Amazonia colombiana. Biomédica. 2002;22:486-98.

Correspondencia: Susan Pillaca Medina Dirección: Condominio los Álamos Dpto. H 204 . Parques del Agustino. El Agustino, Lima, Perú

Teléfono: 9479199944

Correoelectrónico:ssusan_14@yahoo.es 